\title{
种子的长距离风传播模型研究进展
}

\author{
郑景明 桑卫国 马克平* \\ (中国科学院植物研究所植被数量生态学重点实验室 北京 100093)
}

摘 要 植物种子的长距离传播在物种迁移、生物入侵、保护生物学等领域有重要的生态和进化意义。种子传播 有很多方式, 开阔草原等地区的草本植物和许多热带和温带的树木都是通过风传播种子的。风传播的方式最适合 进行种子长距离传播现象的模拟研究。种子的风传播模型是传播生态研究的一个重要领域, 尤其是种子的长距离 风传播模型，对于外来入侵植物的扩散和破碎化景观中植物种群的基因交流等生态过程研究举足轻重，然而国内 鲜见这方面的研究成果。本文综述了种子长距离风传播现象研究的背景和意义, 分析了风传播种子模型的基本形 式和构成原理, 并分别就现象模型和机理模型的相关研究进展进行了总结,同时指出了未来发展的几个重要方向。 种子的风传播模型可以分为现象模型和机理模型两类 现象模型按种子传播核心的形式包括短尾模型、偏峰长尾 模型和混合传播核心模型，后两者对于长距离传播数据的模拟可以取得很好的效果。机理模型按照模拟机制可分 为欧拉对流扩散模型和拉格郎日随机模型两类。本文重点介绍了种子的长距离风传播现象的形成机理和两类机 理模型的参数构成和处理方式。适合种子脱落的天气和适合传播的天气的同步性可能是形成种子长距离风传播 的一个重要前提, 林缘和地表存在的上升气流及大风和暴风中形成的速度梯度都可能对于种子的长距离传播有重 要的作用。机理模型的操作因子主要包括生物方面的因子、气象方面的因子和地形方面的因子。同时对目前几个 应用比较成功的机理模型进行了简要的介绍和评价,包括倾斜羽毛模型、对流-扩散-下降模型、无掩蔽模型、背景模 型、WINDISPER 及其改进模型和 PAPPUS 模型。最后指出, 目前在风传播种子的长距离模型研究中, 对草本植物种 子的传播模拟的投入明显不如树木种子的长距离传播模拟 对于破碎化景观中种子长距离的风传播的研究还存在 很大的差距, 而对提高机理模型预测能力的高分辨率物理环境数据输入技术的需求则为多学科交叉提供了很好的 机会。

关键词 风传播 种子 长距离 模型

\section{ADVANCES IN MODEL CONSTRUCTION OF ANEMOCHORIC SEED LONG-DISTANCE DISPERSAL}

\author{
ZHENG Jing-Ming SANG Wei-Guo and MA Ke-Ping ${ }^{*}$ \\ ( Laboratory of Quantitative Vegetation Ecology , Institute of Botany , Chinese Academy of Sciences , Beijing 100093 , China)
}

\begin{abstract}
Long-distance dispersal (LDD) of plant propagules has significant ecological and evolutionary implications for plant species migrations, biological invasions, conservation biology and many other fields of research. Although seeds can disperse by many different processes, the seeds of many herbaceous species of open grasslands and tree species in temperate and tropic zones are anemochoric, i.e. , wind dispersed. Modeling the dispersal of anemochoric seeds , particularly their long distance dispersal , is a major research field because of the importance of this ecological process for understanding such phenomena as the spread of invasive alien plants and gene flow among meta-populations in fragmented landscapes. Our search results indicated that there are no synthesis papers on the LDD of anemochoric seeds. This paper discusses the background and significance of long-distance dispersal of air-borne seeds, analyzes the basic formulas and structures of models of seed dispersal by wind, summarizes recent advances in phenomenological and mechanistic models, and presents future research directions in this field.

LDD models of anemochoric seeds are categorized into two major classes : phenomenological models and mechanistic models. Phenomenological models include short-tailed dispersal kernels (SDK), Leptokurtic fattailed kernels (LFK) and mixed dispersal kernels (MDK). The LFK and MDK models are most promising for simulating long-distance dispersal of seeds. Mechanical models are categorized into Eulerian advection-diffusion models (EADM) and Lagrangian stochastic models (LSM). The mechanisms of LDD and the major parameters of these two classes of models are a major focus of this paper. Important mechanisms of LDD include
\end{abstract}


synchronization of seed release with suitable weather conditions. and updrafts that occur at forest edges and on the ground surface. Also, gradients of wind speed that form during storms was speculated as being an important mechanism of LDD. Operative factors in wind LDD models include biological , meteorological and topographical factors. We introduce and evaluate a number of models that have been used successfully to model LDD by wind , including tilted plumed model (TPM), advection-diffusion-deposition model (ADDM), no-shelter model (NSM) , background model (BM), WINDISPER , modified WINDISPER (MWINDISPER) and PAPPUS. Lastly , the current status of wind LDD model construction is analyzed and some gaps are pointed out. The authors advocate that more effort should be made to construct models for herbaceous species since currently there are many more wind LDD models for tree species. There are many challenges and needs for modelers to link models with empirical field data of fragmented landscapes. Finally , collaborative approaches among researches from different fields are encouraged in order to improve LDD model forecasting especially with regard to increasing the precision of inputs of attributes of the physical environment.

Key words Anemochoric , Seed , Long-distance dispersal , Model

植物生态学者很早就认识到, 在种子阶段产生 的时空格局对于植物种群的动态十分关键。种子传 播是植物在空间移动的主要阶段, 新个体产生初始 的空间格局, 不仅决定了补充幼苗的潜在范围, 而且 对以后的许多生态过程如捕食和竞争提供了一个模 板最终决定了新的繁殖体的空间格局 (Harper, 1977 ; Howe \& Smallwood, 1982 ; Willson, 1993)。生 态学中有这样的一个假说, 许多植物种在对强竟争 者 (Superior competitor)的竞争中，依赖早传播到达相 对空旷的生境以建立优势 ( Harper, 1977;Holmes \& Wilson，1998）还有假说认为:在斑块环境中种子传 播可以深刻影响种间相互作用, 利于竞争者、捕食者 与猎物、寄生物与寄主的共存从而促进生物多样性 (Opdam , 1990)。许多研究证明 :不同物种的传播能 力的差异是植物群落中多种植物共存的关键 (Waser et al. , 1982 ; Shimida \& Ellener , 1984)。植物种子传 播不仅影响种群的动态和持续生长, 还能改变物种 的丰富度与分布, 进而对群落的结构产生影响。另 外, 作为植物重要的生活史特性, 种子传播对种群的 进化也有复杂的影响，种子传播决定了种群间的基 因流动水平 影响种群的适应、特化和生活史特性的 演化等过程(Dieckmann et al ., 1999 ;Willson \& Traveset , 2000)。

大多数种子只能从种子发散源传播很短距离, 种子密度随距离迅速下降 (Harper, 1977 ; Willson， 1993 )。近 30 余年对种子传播的大量研究也主要集 中在近距离传播 (Short distance dispersal, SDD)上, 如 对逃逸与定居的假说 (Escape and colonization hypotheses)验证多限于小于 $100 \mathrm{~m}$ 的范围内 (McCanny \& Cavers , 1987 ;Hamrick \& Nason , 1996) ;但在种子的传 播过程中，会有少数一些种子可到达很远的地方，即 所谓的种子长距离传播现象 (Long distance dispersal , LDD)。对于近距离传播与长距离传播的划分虽然
没有必要十分明确, 但研究者通常还是有一个约定, 即 LDD 是指从种源开始几百米以上的尺度 (Greene \& Johnson , 1995 ; Cain et al. ,2000)。

\section{生物学家早就认识到植物长距离传播现象的存} 在和潜力。如 Ridley (1930)、 Carlquist (1966)、Sorensen (1986) 都记录了植物种子通过各种途径长距离传播 的情况。Darwin (1959) 指出种子传播的适应与偶然 的种子长距离传播对植物可能很重要, 他根据观察 认为 植物虽然看起来不会动, 但它们有办法跨越很 远距离去开拓新领地。尽管有前人的描述和分析工 作, 但对植物种子的长距离传播研究还是经常被忽 视 或只是在一些研究中以奇闻逸事的方式被提及 (Cain et al. ,2000)。这主要是由于对长距离传播难 以直接跟踪, 通常对于长距离传播的间接证据是通 过远离潜在种源的已经建立的种群 (包括幼苗、幼树 和成熟树)进行的推断, 而没有对种群的长距离传播 事件作记录，因此造成对长距离传播的解释很困难 (Higgins \& Richardson，1999)。植物种子的长距离传 播对种群在岛屿上定居至关重要, 同时对植物种群 在远离大陆的岛屿上定居方面的研究也提供了最多 的长距离传播证据 (Bennett, 1998 ; Cain et al. , 2000)。

关于植物长距离传播的最具学术价值的假说是 Clement Reid 在 1899 年其著作《不列颠植物起源》 (The origin of the British flora)中提出的, 他根据花粉 化石分布的研究证据发现 橡树这一温带物种, 在最 后一次冰期结束到罗马人占领不列颠群岛约 1000 年间，向北扩展了 $1000 \mathrm{~km}$ 到今天的分布领域。尽 管根据过去的记录推断出的快速迁移速率与植物现 在自身的情况吻合很好, 但这种情形却明显与传统 的树木生活史和有限传播的概念中所包括的传播机 理不相符合。其后一个世纪中在北美和欧亚大陆的 类似研究则包括了更多的植物种类, 后人将这种全 
新世时期树种快速迁移的解释中存在的两难情况称 为 Reid 的矛盾 (Reid's paradox) (Clark，1998)。许多 人研究植物的全新世迁移 (Holocene migration) 时, 都 认识到种子长距离传播对植物尤其是树木种类迁移 的重要性 (Skellam,1951; Birks，1989；Wilkinson, 1997 ; Clark , 1998 ; Clark et al ., 1998), 得到的主要 结论是 种子的长距离传播主宰森林下层植物的全 新世迁移活动; 对许多物种而言, 用分层运动模型 (Stratified movement model) 描述物种迁移是合适的 (Cain et al. ,2000)。Reid 也意识到，一定是一些偶 然的长距离定居事件在其中起作用, 他猜想是鸟类 迁徙引起的长距离传播, 而近期有许多直接或间接 的证据证明, 至少对某些树种而言通过风进行长距 离传播是可能的 (Nathan \& Muller-Landau, 2000 ; Horn et al. ,2001)。

虽然植物长距离传播是偶然和稀有的事件 (Cain et al., 2000 ; Nathan \& Muller-Landau , 2000)， 但长距离传播在生态和进化的时空尺度上是异常重 要的, 近期在生物入侵、复合种群动态和保护生物学 等领域的研究中对长距离传播都有所强调。入侵生 物对全球生物多样性造成了严重威胁, 在预测某种 外来种入侵的可能途径时，必须注意到入侵种的长 距离传播能力 (Moody \& Mack, 1988 ; Hengeveld, 1989 ; Shigesada et al ., 1995 ; Higgins et al . , 1996 ; 2000 ; Higgins \& Richardson , 1999) ;在运用模型预测 入侵种对各种环境变化的反应时, 结果的准确性很 大程度上依赖是否在模型中加入了长距离传播 (Dyer , 1995 ; Collingham et al ., 1996 ; Manlanson \& Armstrong , 1996 ; Higgins \& Richardson , 1998 ; 1999)。复 合种群广义上是指一系列空间上间断的种群通过传 播相互联系，一般种群之间的距离为几百米，而植物 种子传播距离一般不超过 $100 \mathrm{~m}$, 因此, 亚种群间的 联系状态取决于长距离传播事件在多大程度上发 生, 由此可见长距离传播对复合种群持续的重要性。 尤其在种子不休眠、生境经常变化或受周期性的毁 灭性干扰的情形下, 长距离传播是种群能够持续下 去的关键性机制, 并有助于在改变的和破碎的生境 中维持复合种群及基因流(Hamrick \& Nason，1996； Cain et al. ,2000)。在保护生物学的文献中, 景观破 碎化导致了种源减少, 而植被斑块间距离增大 种子 长距离传播成为复合种群的持续能力及杂交结果的 重要作用因素 (Neubert \& Caswell, 2000 ; Hewitt \& Kellman , 2002)。

目前长距离传播现象的特殊重要性已经被认识
到 相关的研究也取得了一些理论成果 (Malanson \& Armstrong，1996），但相对种子的近距离传播研究而 言, 对长距离传播的经验性研究还是太少了, 这主要 是由于要数量化地确定稀有事件在技术上存在巨大 的困难 (Greene \& Johnson , 1995 ; Levins , 1995 ; Silvertown \& Charlesworth，2001)。虽然对长距离传播的 定量化研究越来越多, 但在缺少经验数据的情况下, 长距离传播研究取得的进展多数还是在理论上取得 的 模型应用在长距离传播研究已显示其决定性作 用, 因此很多人呼吁加强长距离传播模型方面的研 究(Chambers \& MacMahon , 1994 ; Dyer , 1995 ; Shigesada et al ., 1995 ; Collingham et al ., 1996 ; Clark et al. , 1998 ; Holmes \& Wilson, 1998 ; Higgins \& Richardson , 1999 ; Cain et al. , 2000 ; Clark et al. , 2001 ; Horn et al . , 2001 ; Nathan et al . , 2002 ; Tackenberg，2003; Clark et al. ,2003)。应当指出的是， 许多情况下种子传播是通过多种传播媒体完成的 (Bakker et al ., 1996 ; van Dorp et al , 1996 ; Cain et $a l$ ，２000），也是多阶段完成的（Matlack，1989； Greene \& Johnson, 1997 ; Vander Wall \& Joyner , 1998 ）在种子的各种长距离传播方式中, 凤对种子 的长距离传播是最简单也是最适合模型研究的, 并 且在开阔的草原风是最重要的种子传播载体之一 (van der Pijl , 1982) 许多热带和温带树种的种子也 是以风传播为主 (Greene \& Johnson, 1989 ; Nathan et $a l$. ,2000) ,因此, 采用模型进行种子长距离传播研 究又多集中在风传播种子的模拟方面 (Bennett, 1998 ; Horn et al . 2001) , 尤其是风对树木种子的长 距离传播, 是许多地区森林生态系统过程的重要组 成部分, 目前对森林树种的长距离传播研究是生态 和进化研究的前沿性课题 (Greene \& Johnson , 1995 ; Wilkinson, 1997 ; Clark et al. , 1998 ; Clark , 1998 ; Iverson \& Prasad, 1998)。

\section{1 种子长距离风传播模型的类型}

风播种子模型通常分为两类:现象模型 (Phenomenological model) 和机理模型 (Mechanistic model) (Okubo \& Levin , 1989 ; Nathan et al. ,2002a)。现象 模型主要指传播核心或种子雨的分布拟合函数。传 播的种子围绕其发散源形成的空间分布称种子雨; 通常这一概念用于单株植物为发散源 (点源) 的情况 下，对多株种子发散源(面源)，更常用的概念是传播 核心 (Dispersal kernel), 它的定义是一个描述种子沉 降的位置与种子发散源的关系的概率密度函数，以 
单位面积上种子落地的概率作为与种子发散源的距 离的函数(Nathan et al .,2000)。机理模型对种子传 播的模拟, 是直接通过植物的特性和传播媒体的特 性预测种子传播格局。由于模拟需要包括一些可测 定和解释的因子, 通常需要大量实测数据以确定模 型参数 (Turchin , 1998 ; Nathan et al . 1999)。其优 势在于种子密度随距离的改变是真正可以预测到 的, 而不仅仅是拟合数据。由于所有的参数都对应 真实的研究系统的特征，运行因素(Operative factors) 的变异对种子格局的影响也可以进行检查, 而且总 结的规律可以应用于其它系统 (Nathan et al. , 2000)。

\section{1 种子长距离风传播现象模型}

种子风传播现象模型大致可分成 3 类,短尾模
型(Short-tailed dispersal kernel)、偏峰粗尾模型 (Leptokurtic kernels with fat tails)、混合模型 (Mixed kernel)。早期对种子风传播的模拟主要集中在局部尺 度上,多数采用短尾模型研究种子传播平均距离 (Howe \& Smallwood，1982) 模型主要包括正态分布、 对数正态分布、韦布尔分布、倒幂形曲线、负指数曲 线 (Okubo \& Levin, 1989 ; Higgins \& Richardson, 1999 ;Nathan \& Muller-Landau , 2000 ; Bullock \& Clark , 2000)。随着人们对长距离传播现象重要性的认识, 短尾模型在模拟长距离传播方面显露出明显缺陷, 更多研究提倡采用偏峰粗尾模型和混合模型拟合风 传播种子的近距离传播主体部分和长距离传播部分 (Clark et al. , 1998 ; 1999 ; Bullock \& Clarke , 2000 ; Clark et al .,2001)。主要现象模型及公式见表 1 。

表 1 风传播种子的主要现象模型公式

Table 1 Major phenomenological models and their equations

\begin{tabular}{|c|c|c|}
\hline 模型名称 Model name & 模型公式 Equation & 文献来源 Reference \\
\hline \multicolumn{3}{|l|}{ 短尾模型 Short-tailed dispersal kernels } \\
\hline 负指数模型 Negative exponential model & $f(x)=a e^{-b x}$ & Okubo \& Levin , 1989 ; Bullock \& Clark , 2000 \\
\hline 倒幂函数模型 Inverse power law model & $f(x)=a x^{-b}$ & Bullock \& Clark , 2000 \\
\hline 高斯模型 Gaussian model & $f(r)=\frac{1}{\pi r^{2}} \exp \left[-\left(\frac{r}{a}\right)^{2}\right]$ & Clark et al ., 1998 ; Nathan \& Muller-Landau , 2000 \\
\hline 对数正态模型 Lognormal model & $f(x)=\frac{1}{\sqrt{2 \pi} x \sigma} \exp \left[-\frac{(\ln x-a)^{2}}{2 \sigma^{2}}\right]$ & Greene \& Johnson , 1989 ; Okubo \& Levin , 1989 \\
\hline 韦布尔模型 Weibull model & $f(x)=b x^{c-1} \exp \left[(-b x)^{c}\right]$ & Higgins \& Richardson , 1999 \\
\hline \multicolumn{3}{|l|}{ 偏峰粗尾模型 Leptokurtic fat-tailed kernels } \\
\hline 偏峰粗尾曲线族 Leptokurtic curves with fat tail & $k(x)=\frac{c}{2 \alpha \Gamma(1 / c)} \exp \left[-\left|\frac{x}{a}\right|^{c}\right]$ & Clark et al . , 1998 ; Nathan \& Muller-Landau , 2000 \\
\hline 2Dt 模型 Clark 2Dt model & $f(r)=\frac{p}{\pi u\left[1+\frac{r^{2}}{u}\right]^{p-1}}$ & Clark et al. , 1999 \\
\hline \multicolumn{3}{|l|}{ 混合模型 Mixed dispersal kernels } \\
\hline 混合模型 I Mixed kernel model I & $S_{N}=T_{\dot{A}} \quad\left(\alpha \exp (-b D)+c D^{-p}\right)$ & Bullock \& Clark , 2000 \\
\hline 混合模型 II Mixed kernel model II & $k(x)=p k_{1}(x)+(1-p) k_{2}(x)$ & Clark et al . , 1998 \\
\hline
\end{tabular}

短尾模型中最常用的是倒幂函数曲线和负指数 曲线, 倒幂函数曲线一个优点是可以通过公式两侧 取对数变换成为直线, 因此系数比较好求, 且其参数 无量纲, 可以用于不同尺度的研究, 负指数曲线则通 过一侧取对数可以变成直线形式, 系数也比较好求, 它的一个有意义的优点是当距离趋近 0 时种子密度 值仍为有限大, 因此数学上更易于接受。两种曲线 拟合数据情况相差无几(Fitt et al. , 1987 ; Okubo \& Levin , 1989)。短尾模型的一个主要的缺陷是, 模型 主要应用于短距离传播的拟合, 其分布峰值总是靠 近种源处, 往往与数据不符, 并且都不能表达长距离 传播部分。偏峰粗尾模型是随着对长距离传播的认
识深入而出现的,也是长距离传播理论研究的一个 重要成果(Clark et al，，1998)。近来分析种群传播 的模型开始着重强调传播核心是偏峰核心, 即大多 数种子分布在种子发散源附近聚集, 而曲线的尾部 则延伸很长。应用偏峰核心的模型研究产生的对种 群扩散的一个非直觉的 (Non-intuitive) 结果是种群加 速传播的可能性。按常规扩散形式,种群在传播前 沿以恒定速度前进, 而一个有粗尾的传播核心能够 产生跳跃(Skellam，1951; Hengeveld，1989)。随尾部 的粗度情况不同，种群传播距离与时间的关系可以 是多项式或指数形式(Kot et al. , 1996)。偏峰粗尾 模型存在的一个问题是, 虽然模型有粗尾可以模拟 
长距离传播, 但却往往高估接近种子飞散源部分的 种子密度 (Clark et al. , $1998 ; 2001$ )。进一步的思考 促进 Clark 2Dt 模型的出现, 它们参数较多也就更能 灵活地拟合曲线的任意位置 (Clark et al , , 1999) ,但 同时也增加了模型的复杂程度。混合模型是为了解 决上述这些函数对于同时模拟 SDD 和 LDD 的失败 而出现的，从直觉就可以感到混合模型的优越之处： 它假设种子传播距离的分布是多个统计种群的和， 一部分种群是 SDD, 而另一部分是 LDD。Clark (1998) 为了检验包括长距离传播的模型与传播数据 的一致性, 并评价长距离传播所占的比例 采用了一 个混合核心途径: 传播种子的一部分用参数模拟 局 部”传播; 另一部分模拟 粗尾” 分布, 模型更细致地 包括了近种源和尾部 (LDD) 两个峰形, 拟合数据取 自阿巴拉契亚山脉、内华达山脉、秘鲁的森林树种 (Clark et al ., 1998)。对一些灌木和草本的细小种 子传播的 LDD”研究中, 也采用了混合核心途径, 近 种源处为绝大部分种子的 SDD, 用负指数模型模拟; 远处为数量小但更重要的长距离传播, 用反幂函数 模型拟合 (Bullock \& Clarke, 2000 ; Higgins et al. , 2001)。

采用现象模型可以直接描述种子释放后的种子 密度随距离的渐近式分布关系。现象模型的优势是 简单仅有几个参数, 通常拟合数据也较好。尽管简 单易用, 其不利之处在于其参数不与植物或传播媒 体的特征直接关联。由于只能拟合数据, 不能通过 提前假设并预测, 它不能作为所研究对象以外的系 统的归纳, 对于理解根本机制并将可操作的因素应 用于研究对象以外的物种上去时作用很有限 (Nathan \& Muller-Landau, 2000)。虽然无论哪种现 象模型都可以用于拟合整个传播曲线, 但通常多数 研究只能验证整个分布的中心趋势如峰值 (Greene \& Johnson, 1989; Okubo \& Levin ,1989; Andersen, 1991)。需要对整个分布进行验证的情况多发生在 研究长距离传播的案例中 (Clark et al., 1999 ; Nathan et al. ,2000), 但由于 SDD 占整个传播的绝 大部分, 而且缺少 LDD 经验数据, 尾部无法直接拟 合, 这样研究大多也只能比较一下近距离传播和长 距离传播两部分的拟合适合度, 许多有趣的结论都 是基于短距离范围内的种子收集器的数据上 (Clark et al . , 1999 ; Bullock \& Clarke , 2000)。因此, 虽然由 于种子传播过程的复杂性使得经验模型对于研究长 距离传播有重要的意义,但目前面临的最大困难在 于如何取得需要的数据( Cain et al. , 2000 ; Nathan \&
Muller-Landau , 2000)。

\section{2 种子长距离风传播的机理模型}

计算不同形态种子的下落轨迹的概念模型是 Dinggler 在 1889 年提出的(Burrows，1973），其基本原 理是基于颗粒垂直下落过程中受到的重力、上浮力 和拖曳力作用。对于较重的风传播的种子, 弹道公 式(Ballistic equation) 是最常用来描述种子水平传播 距离的 (Cremer, 1977):

$$
D=\frac{H U}{F}
$$

公式中 $D$ 为种子传播距离, $H$ 为种子释放高 度, $U$ 为水平风速, $F$ 为种子在静止空气中的最终 平衡速度 (Terminal velocity)。Burrows ( $1973 ; 1975$; 1983 ) 分别在此基础上发展了模拟不同风状态的情 况下种子在三维空间中的运动轨迹的模型。随后许 多学者运用它研究不同形态的植物种子风传播的能 力 (Matlack , 1987 ; Hensen \& Muller , 1997) , 并开发出 第一个正式的种子风传播机理模型 SEDFAL (Fields $\&$ Sharpe，1980)和第一个长距离传播模型 SED( Andersen，1991)。弹道公式应用于比较重的风传播种 子, 可以较好地估计种子传播曲线的峰值, 但对于较 轻的种子或传播曲线尾部的模拟则效果不理想 (Greene \& Johnson，1995)。一粒种子的风传播距离 取决于种子的空气动力特征、种子释放高度、飞行过 程中的水平和垂直风速。对种子种群而言, 则应包 括这些参数的分布及种源强度 (Seed source strength, 种子释放的总数) 等, 多项因素共同决定种子传播 曲线 (Greene \& Johnson, 1989; Okubo \& Levin, 1989)。地形因素和气象因素对风的状态参数通常 影响很大, 是建立能准确预测长距离传播的机理模 型所必须重视的 (Higgins et al., 1996 ; Tackenberg, 2003)。种子的长距离传播机理模型根据其模拟途 径可以分为欧拉对流扩散模型 (Eulerian advectiondiffusion models, EADM) 和拉格郎日随机模型 ( ( Lagrangian stochastic models, LSM) 两类 (Jongejans \& Schippers , 1999 ;Nathan 2001)。种子的风传播机理 模型的操作因子主要包括生物方面的因子、气象方 面的因子和地形方面的因子, 机理模型中对主要参 数的一些算法见表 2 。

生物方面的因子主要有种子形态及其空气动力 学特征, 释放高度, 种源强度等。对各种风传播植物 物种的种子的空气特征在实验室有大量详细的研究 (Green ,1980 ; Matlack , 1987 ; Azuma \& Yasuda , 1989 ; Andersen , 1992 ; Andersen , 1993 ; Nathan et al. , 
表 2 机理模型中主要参数和计算方法

Table 2 Major parameters in mechanical models and their treatments

\begin{tabular}{|c|c|c|}
\hline 主要参数 Major parameters & 处理方法 Treatment & 文献来源 Reference \\
\hline \multicolumn{3}{|l|}{ 生物参数 Biological parameters } \\
\hline 释放高度 Height of seed release & 正态分布模型 Normal or measured & Nathan et al ., 2002 ; Tackenberg , 2003 \\
\hline 最终平衡速度 Terminal velocity & 实测 Measured & Nathan et al . , 2002 ; Tackenberg , 2003 \\
\hline \multicolumn{3}{|l|}{ 气象参数 Meteorological parameters } \\
\hline 风向 Wind direction (radians) & 实测 Measured & Nathan et al. , 2001 ; Nathan et al. ,2002 \\
\hline 水平风速 Horizontal wind speed & $\begin{array}{l}\text { 正态分布模型, 对数正态模型 Normal , lognormal, } \\
\text { measured }\end{array}$ & Greene \& Johnson , 1995 ; Nathan et al . , 2002 \\
\hline 水平风速垂直分布 Horizontal wind profile & $\begin{array}{l}\text { 对数模型, 指数模型 幂函数模型 Logarithmic, ex- } \\
\text { ponential , power law }\end{array}$ & $\begin{array}{l}\text { Greene \& Johnson , } 1995 \text {; Nathan et al . , } 2001 \text {; } \\
\text { Tackenberg , } 2003\end{array}$ \\
\hline 垂直风速 Vertical wind speed & 正态分布模型 ,实测 Normal distribution or mesured & $\begin{array}{l}\text { Greene \& Johnson , } 1995 \text {; Nathan et al . , } 2002 \text {; } \\
\text { Tackenberg , } 2003\end{array}$ \\
\hline 上升气流速度 Updraft by turbulence & 实测 Measured & Tackenberg , 2003 \\
\hline \multicolumn{3}{|l|}{ 地形参数 Topographical parameters } \\
\hline 取代高度 Replacement height & 线形模型，实测 Linear or measured & Nathan et al ., 2002 \\
\hline 粗粘长度 Roughness length & 线形模型, 实测 Linear or measured & Nathan et al ., 2002 ; Tackenberg , 2003 \\
\hline 地形高度 Height of position & 线形模型，实测 Linear or measured & Nathan et al. , 2001 ; Tackenberg , 2003 \\
\hline
\end{tabular}

2000 ; Yasuda \& Azuma, 1997 ; Tackenberg et al. , 2003 ), 但在野外研究较少 (Greene \& Johnson , 1980 ; Augspurger \& Franson , 1987 ; Tackenberg , 2003)。风 传播的种子有各种各样的形态特征利于其飞行,如 种翅、冠毛等，虽然各种形态的种子飞行中作用机理 不一样，但表现出的飞行结果却都很相似(Burrows， 1973 ; Augspurger , 1986 ; Matlack ,1987)。种子下落 过程中的最终平衡速度被认为是最重要的空气动力 特征, 常常用做传播能力的替代物, 因为它反映了种 子被风带走的时间（Green，1980; Burrows，1983; Andersen , 1991; 1993)。这一特性主要受繁殖体的 质量和表面积的影响，与翅载力 (Wing-loading, 定义 为重量与表面积之比) 高度相关 (Norberg , 1973 ; Green , 1980 ; Augspurger \& Franson, 1987)。测定最 终平衡速度的方法主要有下落法、漂浮法 (Nathan $e t$ al. , 2001 ; Jongejans \& Schippers , 1999 ; Tackenberg, 2003)。种子释放高度直接决定了种子在空中停留 的时间, 是影响传播距离的另外一个重要的因子。 对于草本植物和灌木, 它的作用要比乔木大 ; 要比较 真实地模拟植物的种子传播距离, 就必须考虑植物 的形态和种子的垂直方向的分布对种子传播核心的 影响。一般点源种子释放模拟时, 种子在植株上的 分布高度通常通过球面、锥体等模拟 或按经验确定 为一定高度范围上的随机分布 (Greene \& Johnson, 1995 ; Nathan et al., 2002 ; Cousens \& Rawlinson, 2001 ; Horn et al . ,2001)。

气象因子方面风的因素是最重要的, 对水平和 垂直方向上风速变化模拟方面研究是提高整个模型
的模拟精度的关键。因为水平风速应当是随时空而 变不可能是恒定的数值, 时间上水平风速的变化一 般通过实际测定或引用实验地附近的气象站的数据 进行转化 (Greene \& Johnson, 1995 ; Nathan et al. , 2001 ; Tackenberg，2003）,简化的处理可采用韦布尔 分布模拟 (Andersen，1991)。通常假定种子飞行过 程中初始水平风速只随高度变化而同一高度不变, 因此一个重要的问题是不同高度上风速的分布模 拟。许多研究认为用对数模型描述多种植被类型上 面风速分布效果都很好 (Monteith \& Unsworth，1990； Wieringa，1993)。但森林上空或空地上与森林内部 的水平风速显然不同, 多数风传播种子模型采用对 数模型模拟空地的水平风速分布, 而在森林内则需 用其它曲线，如指数或幂函数形式(Fields \& Sharpe， 1980 ; Greene \& Johnson, 1989 ; Greene \& Johnson , 1995 ; Nathan et al . 2002b ;Nathan et al . 2002)。而 垂直方向上的风速只能够采用三向风速仪测定, 虽 然一般气象站并不记录此类数据, 但是对于长距离 传播的模拟涉及到极少量因为垂直风速的变异引起 的偶然传播事件，简化的分析计算往往会造成很大 的误差, 因此垂直风速的测定是必须的 (Greene \& Johnson , 1995 ; Nathan et al. 2001 ; Higgins et al. , 2001 ; Tackenberg, 2003)。但对于一些较重的树木 的种子, 有的模型忽略垂直风速已简化模拟过程提 高模型的通用性 (Greene \& Johnson , 1989) , 也有的模 型为了特定的模拟目的假定垂直风速永远小于种子 的最终平衡速度 (Nathan et al . , 1999), 但对较轻的 种子这些假定都是不可接受的，因为此时涡流作用 
比种子自身的最终平衡速度的影响要大, 垂直方向 的风速变异是关键的模型运行因子 (Okubo \& Levin , 1989)。对种子风传播的模型总结表明 :更接近真实 情况的风传播种子的机理模型还必须考虑地形等因 子对风的影响及风有关的各参数的变异, 尤其是垂 直风速参数的变异, 因为各种参数变异的累计是导 致一些偶然事件如长距离传播发生的前提 (Augspurger , 1986 ; Greene \& Johnson , 1989 ; Andersen , 1991 ; Nathan et al ., 2001 ; Tackenberg , 2003)。

地形因子包括海拔高度, 坡度, 地表摩擦力情况 等, 对种子的传播距离有相当大影响, 尤其下降速度 较小的种子 忽视地形可能导致极大地低估种子的 风传播能力。举个简单的例子, 如果一个草本植株 位于很高的陡坡上面, 那么在同样的气象条件下它 的种子将比平地植株的种子飞得更远。有研究发现 甚至很小的高程和坡度差异都会导致长距离种子的 比例增高很多 (Tackenberg，2003)。然而, 很多风传 播种子模型对地形因素都过度简化甚至忽略了, 这 直接导致对种子传播能力的低估 (Greene \& Johnson , 1995 ; Nathan et al. ,2002b ; Tackenberg , 2003)。对 此方面因素的模拟目前进展不大。

\section{2 种子的长距离风传播机理}

虽然决定种子传播距离谱 (Seed dispersal distance spectra)和全部种子中长距离传播比例的因素 很多,但影响风传播种子传播距离的因素中，气象因 素, 尤其是风的作用是最基本也是最重要的, 其重要 性往往超过种子本身的生物学特性 (Greene \& Johnson , 1995 ; Nathan et al . 2002a ; Tackenberg , 2003)。 如果要更好地模拟长距离传播, 必须仔细考虑气象 因子对种子的种种影响。气象因素可能在以下几个 方面促进长距离传播的形成。

首先种子脱离植株需要的条件与气象条件的关 系密切。有许多研究表明风向与风速与某类植物种 子的脱落数量有关联, 并且脱离风速基本在季节和 每天平均风速范围内 (Augspurger，1986)。例如, 针 叶树种子释放与相对湿度有关而与风速低关联,6 种阔叶树的翼果最低脱离风速约 $2 \mathrm{~m} \mathrm{~s}^{-1}$ (Matlack, 1987 ; Nathan et al . 2000) ;几种桦树种子往往在最 适合长距离风传播的情况下脱落，因其分离层在湿 度很低的情况下发育更快, 而一天中湿度最低时间 在中午后下午初, 而此时风一般最大, 热对流单元也 最易形成（Ford et al.，1983；Greene \& Johnson， 1992)。发现某些树种的种子脱落概率大致与拖曳
力 (即水平风速的平方) 成正比, 脱落风速为平均风 速的 1.8 倍左右, 向上的风比水平方向上的同等速 度的风更能使种子有效地脱离植株，说明种子自然 释放也偏爱上升气流 (Ford et al . , 1983 ; Greene \& Johnson, 1995)。几种乔木的翼果易于在早秋的大 风和后期的中等风中脱落飞行(Horn et al . ,2001)。 空气的干燥程度也会影响种子的脱落, 许多植物在 干燥的天气种子脱落, 这种现象叫干裂 (Xerochasy) (Burrows，1973 ; van der Pijl，1982)，而向上的热气流 也比较容易在晴朗而干燥的天气下形成 (Tackenberg，2003）这种同步性可能是形成长距离传播的 一个重要条件。因此气象因素对于种子的长距离传 播很重要, 适合脱落的天气和适合传播的天气的同 步性可以提高对种子长距离传播的预测能力 (Nathan et al. , 2001 ; Tackenberg , 2003)。

其次，对一些森林中乔木的种子 林缘垂直风速 大于种子下降速度时, 可使种子脱离林冠层向上, 进 入高空对流层然后长距离传播。以林冠层高度为 界 林冠层下方水平风速近乎指数形式; 而林冠层上 方则接近对数形式, 在林冠层高度, 风速梯度最大， 向上的气流导致种子上升进而长距离传播。因此风 传播种子也就成为两个阶段, 近林缘处水平风速极 低,形成了强烈的种子密度随距离下降的趋势 (Nathan et al ，2001)。在远距离处，极端的垂直风 速 (高速上升气流) 起主要作用, 导致相对很少的种 子沉降 (Horn et al. , 2001)。在林冠层附近剪切力 产生的涡流是产生上升力的机理, 而且此时的瞬时 风速轮廓图决非平均情况那样, 近期用高频三向超 声风速仪测定发现，在林冠层附近阵风可以偶尔产 生 $2 \mathrm{~m} \mathrm{~s}^{-1}$ 的垂直风速（Katul \& Chang，1999），这种情 况下各种子的命运不同，上升的种子中，有可能成为 长距离传播者有机会进入景观尺度上的热上升气流 中，从而可能飞行很远的距离 (Horn et al. ,2001)。 采用相关模型的研究也支持这一观点 (Greene \& Johnson , 1995 ; Tackenberg , 2003)。

再者，对一些草本或比较轻的种子，地表产生的 向上热气流也可以将下降速度较小的种子等带到数 千米高空, 从而使一部分种子长距离传播 (Solbreck \& Andersson , 1987)。根据一年中实际测定的垂直风 速发生频率分析, 在开阔平地最大平均垂直风速分 别为 $1.4 \mathrm{~m} \mathrm{~s}^{-1}$ (5 s 平均)、 $0.8 \mathrm{~m} \mathrm{~s}^{-1}$ (10 s 平均)、 $0.6 \mathrm{~m} \mathrm{~s}^{-1}$ (60 s 平均) (Tackenberg, 2003)。同时可能 发生的另一种情况是, 上升气流的存在导致种子空 中漂浮 种子始终下降但下降的速度很慢, 使有的种 
子有机会飞行很远距离。如一个树木种子传播机理 模型 WINDISPER 虽然假定种子的向上速度总是小 于种子的最终平衡速度的限制，但模拟结果仍有长 距离传播发生, 如 100000 次模拟中, 有 $3.5 \%$ 的种 子飞行距离超过 $100 \mathrm{~m} ; 0.2 \%$ 种子飞行距离超过 $1000 \mathrm{~m}$,最大种子传播距离为 $216 \mathrm{~km}$ ( Nanthan $e t$ al. , 2001)。

还有一个有关种子长距离传播的机理假说可以 极大地提高种子在大风和暴风中的传播范围, 即种 子在大风和暴风中的所谓伯努里航行 (Bernoulli sailing)。大风和暴风中有许多小的速度梯度, 从而产 生伯努里压力, 使种子进入更高的风速中。计算表 明, 大约 $2 \mathrm{~cm}$ 距离上有 $5 \sim 10 \mathrm{~m} \mathrm{~s}^{-1}$ 的速度梯度, 即 可以使一些树木的翼果发生伯努里效应 (Horn et $a l$ ，2001)。虽然有人也曾提出相似说法(Hensen \& Muller, 1997) ,但这个说法尚末证实,也没有已发表 的实验结果。

\section{3 几个值得注意的种子长距离风传播机理 模型}

空气中的粒子传播过程可以模拟为拉格郎日传 播过程或欧拉传播过程，相应的模型可以分为欧拉 对流扩散模型和拉格郎日随机模型。种子的长距离 传播机理模型的模拟途径可以大致分为这两类 (Jongejans \& Schippers , 1999 ;Nathan ,2001)。欧拉对 流扩散模型又可称羽毛模型 (Plume models) (Tackenberg，2003），主要依据扩散原理通过浓度梯度传播 进行种子群体扩散的模拟, 种子到达一定距离的概 率通过部分差分方程计算, 这些方程的解需要有一 些特定关闭假设 ( Ad hov closure assumptions) (Ley \& Thomson，1983；Andersen，1991），而对于复杂情况， 如对流上升气流 (Convective updraft) 存在时, 则很难 做出这种假设。因此用于种子的羽毛模型没有包含 上升气流，从而也就不能对长距离传播做出可靠的 估计(Walklate , 1987 ; Andersen , 1991 ; Wilson \& Sawford, 1996 ; Bullock \& Clarke, 2000 ; Nathan et al. , 2001)。而拉格郎日粒子传播模型是通过对每一个 颗粒物质的飞行过程的模拟实现对全部颗粒物质的 传播模拟。拉格郎日随机模型又称自由行走模型 (Random walk models) (Andersen, 1991 ; Greene \& Johnson, 1995)。主要方法是计算每一个种子在极 小时间内的移动并累积, 表现出飞行的路径。它的 优势是避免了特定关闭假设, 因此可以用于比较复 杂的环境中，而且算法清晰(Ley \& Thompson , 1983)。
拉格郎日模型可以比欧拉模型包括更多的涡流特 性，因为欧拉模型的梯度转移的假定，妨碍它在比典 型浓度扩散尺度更大的尺度上的应用 相对而言，拉 格郎日模型更灵活 概念简单，在适当粒子数量情况 下，计算花费更小(Wilson \& Sawford，1996； Tackenberg , 2003)。

Okubo 和 Levin (1989) 提出了模拟种子和花粉 两者的种子长距离风传播的欧拉模型——倾斜羽毛 模型 (Tilted plumed model) 和对流、扩散和下降模型 (Advection-diffusion-deposition model)。羽毛模型主要 是在气体中的悬浮微粒扩散或空气污染的背景下建 立的, 主要用于描述烟筒排放的气体污染物的扩散 情况(Turchin，1998)，因此比较注意悬浮物的主体 部分而不大在意传播核心的尾部; 其次,它只能用于 很轻的颗粒，不考虑重力下沉作用，对下降速度非零 的粒子, 通常比悬浮物受到向上气流的影响更大 (Walklate，1987；Wilson \& Sawford，1996)。倾斜羽 毛模型是在高斯羽毛模型 (Gaussian plume models) 基 础上改进的 模型主要考虑空气中种子的浓度到地 面浓度是如何扩散的，描述种子在顺风方向和垂直 风方向两种扩散过程。另一个更有希望的模型是在 倾斜羽毛模型基础上提出的对流、扩散和下降模型。 在该模型中, 浴流作用实际是减少了种子传播的中 值距离，与 Burrows (1975; 1983)的研究结论一致。同 时种子传播距离的方差增大, 使得长距离传播成为 可能。

Greene 和 Johnson( 1989)提出了一个带翅或冠毛 的种子的微气象模型 (Micro-meteorology model) ,包括 6 个可测定参数, 平均释放高度、平均种子最终平衡 速度、种子最终平衡速度的标准差, 垂直风速的标准 差, 平均水平风速, 水平风速对数的标准差。其模拟 基础也是对流和扩散动态，包括涡流的效应，主要对 短距离种子进行模拟，结果发现对传播峰值的估计 偏低。Greene 和 Johnson(1995) 将该模型扩展去说明 长距离传播,在其无掩蔽模型 (No-shelter model) 中, 更多的种子在高风速条件下脱离植株。他们同时还 提出了另一个模型, 称为背景模型 (Background model) 其中假定长距离传播的种子是那些正在向垂直 风速大于种子的最终平衡速度的向上气流作用下扩 散的种子，这份种子比例的估计，在北美 代表性”风 播种子的树种中为 $0.1 \% \sim 10 \%$ 。他们还用前人和 自己的种子捕捉数据对两个模型进行了验证，结果 表明两个模型对长距离传播的预测能力一般偏小。 对在 500 1500 m 范围种子密度的预测, 作者更认 
可背景模型预测得到的种子传播密度随距离稳定的 结果而不是随距离种子密度下降的结果 (Greene \& Johnson，1995)。此后他们对模型作了进一步修正， 修改后的模型对于长距离传播的预测能力得到很大 提高( Tackenberg，2003)。

Nathan 等(2000)采取拉格郎日途径建立了一个 机理模型 WINDISPER。该模型整合水平风速、垂直 风速、种子最终平衡速度、释放高度的随机效应, 模 拟每一粒种子的传播。对每一种子，运算的因子来 自该参数实测的分布值，该模型对地中海松 (Pinus halepensis)林周围, 距离最近植株 $110 \mathrm{~m}$ 范围以 $1 \mathrm{~m}^{2}$ 的空间分辨率可信地 $(R=0.80)$ 预测了其种子传播 密度, 时间格局主要体现为一定期间的种子产量和 风速情况，主要依据气象参数计算，从而决定种源力 和种子的飞行距离等(Nathan et al. ,2001)。模型有 一个特征对长距离传播背景而言比较重要, 即假定 垂直风速恒小于种子最终平衡速度以保证种子的有 限降落距离。这样条件下,仍然可以预测到几百公 里的距离, 因为可能出现极小的垂直风速-种子最终 平衡速度组合与相对较大的水平风速和释放高度。 因此，该模型可以作为没有向上气流作用时的长距 离传播事件发生的参考, 也可以修改上面的假定进 一步模拟长距离传播。Nathan 等 (2002a) 进一步耦 合拉格郎日途径和欧拉途径, 结合冠层附近的涡流 生成模拟模型，建立了新一代模型 MWINDISPER (Modified WINDISPER), 并用于预测 5 个温带森林和 北方阔叶林的重要风传播树种的种子扩散，结果发 现 ,多数种子传播距离在 $10^{2} \mathrm{~m}$ 尺度范围内; 而在有 上升气流的情况下, 有 1\% 5\% 的种子可以传播到 $10^{4} \mathrm{~m}$ 尺度的距离 (Nathan et al .,2002a)。

Tackenberg (2003) 针对欧洲中部的风传播的草 本植物建立了一个拉格郎日模型 PAPPUS，他注意到 现有的风传播模型一般都对植物的传播能力估计过 低的问题，在模型建立过程中充分考虑地形和上升 气流对种子长距离传播的影响, 通过引入涡流模块 和景观特征模块, 比较创新地解决了这两方面的缺 陷。PAPPUS 对于种子下降速度在 $0.15 \sim 0.9 \mathrm{~m} \mathrm{~s}^{-1}$ 的种子都适用, 也就是说几乎所有草本植物都可以 模拟。在各种天气和地形进行 13 次释放实验得到 的 $>100 \mathrm{~m}$ 的长距离传播种子比例数据与模拟结果 都没有显著差异。PAPPUS 不但预测到长距离传播 比例, 而且预测到了传播距离谱的双峰形式 ( Tackenberg，2003)。但模型中的涡流和热上升气流并不 是通过计算得到, 而是采用对风媒介的直接高频测
定值 地形方面的改进, 是在地图栅格化后, 采用数 字化高程模型和粗粘长度图来为两个景观参数高程 和地面粗䊁长度赋值。在这方面都还有很大改进的 余地。

\section{4 种子长距离风传播模型的研究方向}

种子长距离传播模型的研究, 当研究背景为气 候变化与物种迁移关系时, 更多的是研究乔木树种 的种子扩散 (Clark , 1998 ; Clark et al . , 1999 , 2001 ; Greene \& Johnson , 1989 ; 1995 ; Manlanson \& Armstrong , 1996 ; Manlanson \& Cainns , 1997 ; Vander Wall \& Joyner , 1998 ; Nathan et al . ,2000 ;2001 ;2002) 极 少有对草本的研究 (Cain et al. , 1998 ; Bennett, 1998 ; Bullock \& Clarke ,2000 ; Tackenberg ,2003)。原 因之一是，同乔木的风传播的丰富的孢粉化石相比， 草本一般花粉多为昆虫传播, 化石记录中孢粉数量 相对稀少, 因而对草本物种长距离传播现象的理论 研究很困难。而在模型建设过程中，树木种子比较 大无疑比较容易取得野外数据以确定模型相关参 数, 所以导致有关草本种子的长距离传播模型方面 的研究相对较少。但重要的一点是, 长距离传播现 象不仅在全新世树木迁移研究中存在, 它对植物生 物学研究很多关键方面都有重要的影响,包括种群 动态、种群进化、复合种群动态、生物入侵、生态群落 的动态和多样性(Cain et al ., 1998; 2000)。因此对 于草本种子长距离传播模型的研究与森林物种的研 究同样重要, 同样有挑战性和吸引力, 必将成为将来 的一个重要研究方向。

全球变化改变了植物的生境, 未来的植物多样 性分布和相应生态系统功能的变化很大程度上决定 于植物如何迁徙到适合的生境 (Pitelka et al. , 1997)。然而人为活动造成现实生境的一个重要特 征是异质性，即包括生境丧失和破碎景观，因此特别 缺乏的是一种联系, 将长距离传播现象模型与描述 传播过程与异质性环境相互作用结果连接起来。影 响种子传播距离的过程很多样, 通常是各种生态环 境下有各自的特殊性，而且人们对此知之甚少 经验 性模型的研究也是一种比较实用的做法。将现有的 关于长距离传播的现象模型成果与描述影响种子在 破碎的景观中运动的方程连接, 可以描述更为真实 的植物传播图景, 这方面的研究虽然已经逐渐开展, 但研究力量仍显不足, 是将来长距离传播模型发展 的一个重要方向, 也是一个巨大的挑战 (Higgins \& Richardson , 1999 ; Cain et al . , 2000 ; Higgins \& Cain , 
2002 ; Higgins et al. , 2003)。

建立长距离传播机理模型, 涡流对于种子长距 离传播发生至关重要。在高强度的涡流发生情况 下, 大气边界层中底层颗粒物质可以上升到表层的 顶部，从而达到具有相当高的水平和垂直风速的高 度则种子长距离传播才能成为现实 (Oke，1987)。 虽然已经有研究在长距离传播模型中包括了地形和 热流引起的涡流和对流的效应, 但其存在的局限性 则有待进一步克服和改善 (Horn et al ., 2001; Nathan et al , 2002; Tackenberg，2003)。为了产生 可信的计算结果, 长距离传播模型应用高的时空分 辨率数据输入, 以充分解决相关的物理过程; 应用大 涡旋模拟技术 (Large-Eddy Simulation) 为传播模型产 生输入数据, 越来越受到重视。有人将大涡流模拟 模型与拉格郎日粒子传播模型耦合, 研究在不同类 型的景观中的被动示踪物的传播 (Avissar et al. , 2001）虽然至今还没有正式发表的研究结果，但这 种前景都在鼓舞着更多研究者, 希望能将这种大尺 度的气象现象纳入种子风播模型，从而能更准确地 实现长距离传播的模拟和预测。采用更多的其它学 科的技术和手段肯定有助于解决长距离传播的模拟 问题, 因为在传播生态学领域, 多学科交叉研究必将 产生丰厚的回报 (Nathan，2001)。

\section{参 考 文 献}

Andersen, M. C. 1991. Mechanistic models for the seed shadows of wind-dispersed plants. The American Naturilist, 137:476 497.

Andersen, M. C. 1992. An analysis of variability in seed settling velocities of several wind-dispersed Asteracea. American Journal of Botany, 79: $1087 \sim 1091$.

Andersen, M. C. 1993. Diaspore morphology and seed dispersal in several wind-dispersed Asteracea. American Journal of Botany, 80: $487 \sim 492$.

Augspurge, C. K. 1986. Morphology and dispersal potential of wind-dispersed diasporas of neotropical trees. American Journal of Botany, 73:353 363.

Augspurger, C. K. \& S. E. Franson. 1987. Wind dispersal of artificial fruits varying in mass, area, and morphology. Ecology, 68: $27 \sim 42$.

Avissar, R., R. E. Walko, S. Thomas, S. B. Roy, G. Katul, R. Nathan, H. Horn \& S. Levin. 2001. A Large-Eddy/Lagrangian Dispersion model to study long-distance dispersal of tree seeds. In: Ecological Society of America ed. Symposium abstracts of the 86th annual meeting of the Ecological Society of America. Washington D. C. : Washington University Press. 6 7.

Azuma, A. \& K. Yasuda. 1989. Flight performance of rotary seeds. Journal of Theoretical Biology, 138:23 53 .

Bakker, J. P., P. Poschlod, R. J. Strykstra, R. M. Bekker \& K. Thompson. 1996. Seed banks and seed dispersal: important topics in restoration ecology. Acta Botanica Neerlandica, 45:461 $\sim 490$.
Bennett, K.D. 1998. The power of movement in plants. Trends of Ecology and Evolution, 13:339 340.

Birks. H. J. B. 1989. Holocene isochrone maps and patterns of tree-spreading in the British Isles. Journal of Biogeography, 16: $503 \sim 540$.

Bullock, J. M. \& R. T. Clarke. 2000. Long distance seed dispersal by wind: measuring and modeling the tail of the curve. Oecologia, 124:506 521.

Burrows, J. M. 1973. Calculation of the primary trajectories of plumed seeds in steady winds with variable convection. New Phytologist, $72: 647 \sim 664$.

Burrows, J. M. 1975. Wind-borne seed and fruit movement. New Phytologist, 75:405 418.

Burrows, J. M. 1983. Calculation of the primary trajectories of plumed seeds and other particles in strong winds. Proceedings of the Royal Society of London, A389: $15 \sim 66$.

Cain, M. L., B. G. Milligan \& A. E. Strand. 2000. Long-distance seed dispersal in plant populations. American Journal of Botany, 87: $1217 \sim 1227$.

Cain, M.L., H. Damman \& A. Muir. 1998. Seed dispersal and the Holocene migration of woodland herbs. Ecological Monographs, 68:325 347 .

Carlquist, S. 1966. The biota of long-distance dispersal. I. Principles of dispersal and evolution. Quarterly Review of Biology, 41: $247 \sim 270$.

Chambers, J.C. \& J. A. MacMahon. 1994. A day in the life of seed: movements and fates of seeds and their implications for natural and managed ecosystems. Annual Review of Ecology and Systematics, 25:263 392 .

Clark, J. S., M. Levwis, J. S. MCLachlan \& J. H. Lambers. 2003. Estimating population spread: what can we forcast and how well. Ecology, 84:1979 1988.

Clark, J. S. 1998. Why trees migrate so fast: confronting theory with dispersal biology and the paleorecord Invasion. The American Naturalist, 152:204 224 .

Clark, J. S., C. Fastic, G. Hurtt, S. T. Jackson, C. Johnson, G. A. King, M. Lewis, J. Lynch, S. Pacala, C. Prentice, E. W. Schupp, T. Webb III \& P. Wychoff. 1998. Reid's paradox of rapid plant migration - dispersal theory and interpretation of paleoecological records. BioScience, 48:13 24 .

Clark, J. S., M. Lewis \& L. Horvath. 2001. Invasion by extremes: population spread with variation in dispersal and reproduction. The American Naturalist, 157:537 554 .

Clark, J. S., M. Silman, R. Kern, E. Macklin \& J. HilleRisLambers. 1999. Seed dispersal near and far: pattern across temperate and tropical forests. Ecology, 80:1475 1494 .

Collingham, Y.C., M. O. Hill \& B. Huntly. 1996. The migration of sessile organisms: a simulation model with measurable parameters. Journal of Vegetation Science, 7:831 846 .

Cousens, R. D. \& A. A. Rawlinson. 2001. When will plant morphology affect the shape of a seed dispersal "kernel"? Journal of Theoretical Biology, 211:229 238 .

Cremer, K. W. 1977. Distance of seed dispersal in eucalyptus estimated from seed weights. Austrilian Journal of Forest Research, $7: 225 \sim 228$.

Darwin, C. 1859. The origin of species. London: John Murray. $323 \sim 335$.

Dieckmann, U., B. O'Hara \& W. Weisser. 1999. The evolutionary ecology of dispersal. Trends in Ecology \& Evolution, 14:88 $\sim 90$.

Dyer, J. M. 1995. Assessment of climate warming using a model of 
forest species migration. Ecological Modelling, 79:199 219 .

Fields, D.E. \& D. M. Sharpe. 1980. SEDFAL: a model of dispersal of tree seeds by wind. Oak Ridge National Laboratory Report, $80 \sim 87$.

Fitt, B.D.L., P.H. Gregory, A.D.Todd, H.A. McCartney \& O. C. MacDonald. 1987. Spore dispersal and plant disease gradients: a comparison between two empirical models. Journal of Phytopathology, 118: $227 \sim 242$.

Ford, R.H. 1985. Life history strategies in two co-existing agamospecies of dandelion. Biological Journal of the Linnean Society, 25: $169 \sim 186$.

Ford, R.H., T.L. Sharik \& P.P. Feret. 1983. Seed dispersal of the endangered Virginia round-leaf birch (Betula uber). Forest Ecosystem and Management, 6:115 128 .

Ghersa, C. M. \& M. L. Roush. 1993. Searching for solution to weed problems. Bioscience, 43: $104 \sim 109$.

Green, D.S. 1980. The terminal velocity and dispersal of spinning samaras. American Journal of Botany, 67:1218 1224.

Greene, D.F. \& E. A. Johnson. 1989. A model of wind dispersal of winged or plumed seeds. Ecology, 70:339 347 .

Greene, D.F. \& E. A. Johnson. 1995. Long-distance wind dispersal of tree seeds. Canadian Journal of Botany, 73:1036 1045.

Greene, D.F. \& E. A. Johnson. 1997. Secondary dispersal of tree seeds on snow. Journal of Ecology, 85:329 340 .

Greene, D. F. \& E. A. Johnson. 1992. Fruit abscission in Acer saccharinum with reference to seed dispersal. Canadian Journal of Botany, 70:2277 2283.

Hamrick, J. L. \& J.D. Nason. 1996. Consequences of dispersal in plants. In: Rhodes, O.E., R. K. Chesser \& M. H. Smith eds. Population dynamics in ecological space and time. Chichago: The University of Chicago Press. $203 \sim 226$.

Harper, J. L. 1977. Population biology of plants. London: Academic Press. $12 \sim 15$.

Hengeveld, R. 1989. Dynamics of biological invasions. London: Chapman \& Hall. $1 \sim 29$.

Hensen, I. \& C. Muller. 1997. Experimental and structural investigations of anemochorous dispersal. Plant Ecology, 133: 169 180 .

Hewitt, N. \& M. Kellman. 2002. Tree seed dispersal among forest fragments. II. Dispersal abilities and biogeographical controls. Journal of Biogeography, 29:351 363 .

Higgins, S. I. \& M. L. Cain. 2002. Spatially realistic plant metapopulation models and the colonization-competition trade-off. Journal of Ecology, 90:616 626.

Higgins, S.I., D. M. Richardson \& R. M. Cowling. 2001. Validation of a spatial simulation model of a spreading alien plant population. Journal of Applied Ecology, 38:571 584 .

Higgins, S.I., S. Lavorel \& E. Revilla. 2003. Estimating plant migration rates under habitat loss and fragmentation. Oikos, 101 : $354 \sim 366$

Higgins, S. I. \& D. M. Richardson. 1998. Pine invasions in Southern hemisphere: modeling interactions between organism, environment, and disturbance. Plant Ecology, 135:79 93.

Higgins, S. I. \& D. M. Richardson. 1999. Predicting plant migration rates in a changing world: the role of long-distance dispersal. The American Naturalist, 153:464 475 .

Higgins, S. I., D. M. Richardson \& R. M. Colwing. 1996. Modeling invasive plant spread: the role of plant-environment interactions and modeling structure. Ecology, 77: $2043 \sim 2054$.

Higgins, S. I. , D. M. Richardson \& R. M. Cowling. 2000. Using dynamic landscape model for planning the management of alien plant invasions. Ecological Applications, 10:1833 1848 .

Holmes, E. E. \& H. B. Wilson. 1998. Running from trouble: longdistance dispersal and the competitive coexistence of inferior species. The American Naturalist, 151:578 586 .

Horn, H. S., R. Nathan \& S. R. Kaplan. 2001. Long-distance dispersal of tree seeds by wind. Ecological Research, 16:877 885 .

Howe, H. F. \& J. Smallwood. 1982. Ecology of seed dispersal. Annual Review of Ecology and Ecosystems, 13:201 228 .

Iverson, L. R. \& A.M. Prasad. 1998. Predicting abundances of 80 tree species following climate change in the eastern United States. Ecological Monograph, 68:465 485.

Jongejans, E. \& P. Schippers. 1999. Modelling seed dispersal by wind in herbaceous species. Oikos, 87:362 372 .

Katul, G. G. \& W. H. Chang. 1999. Principle length scales in second-order closure models for canopy turbulence. Journal of Applied Meterology, 38:1631 1643 .

Kot, M., M. A. Lewis \& P. van den Driessche. 1996. Dispersal data and the spread of invading organisms. Ecology, 77:2027 2042 .

Leishman, M. , L. Hughes, K. French, D. Amstrong \& M. Westoby. 1992. Seed and seedling biology in relation to modeling vegetation dynamics under global climate change. Austrialian Journal of Botany, 40:599 613.

Levins, R. 1995. Toward an integrated epidemiology. Trends in Ecology \& Evolution, 10:304 305 .

Ley, A. \& D.J. Thomson. 1983. A random walk model of dispersion in the diabatic surface layer. Quaterly Journal of the Royal Meterological Society, 100:847 880.

Malanson, G.P. \& D. M. Cairns. 1997. Effects of dispersal, population delays, and forest fragmentation on tree migration rates. Plant Ecology, 131:67 79 .

Malanson, G.P. \& M. P. Armstrong. 1996. Dispersal probability and forest diversity in a fragmented landscape. Ecological Modelling, 87:91 102.

Matlack, G. R. 1987. Diaspore size, shape, and falling behavior in wind-dispersed plant species. American Journal of Botany, 74: $1150 \sim 1160$.

Matlack, G. R. 1989. Secondary dispersal of seed across snow in Betula lenta, a gap-colonising tree species. Journal of Ecology, 77: $853 \sim 869$.

McCanny, S.J. \& P. B. Cavers. 1987. The escape hypothesis: a test involving a temperate annual grass. Oikos, 49:67 76 .

Monteith, J.L. \& M. H. Unsworth. 1990. Principles of environmental physics. London: Edward Arnold Press. $77 \sim 89$.

Moody, M. E. \& R. N. Mack. 1988. Controlling the spread of plant invasions: the importance of nascent foci. Journal of Applied Ecology, 25:1009 1021 .

Nathan, R. \& H. C. Muller-Landau. 2000. Spatial patterns of seed dispersal, their determinants and consequences for recruitment. Trends in Ecology and Evolution, 15:278 285 .

Nathan, R. 2001. The challenges of studying dispersal. Trends in Ecology \& Evolution, 16:481 483.

Nathan, R., G. G. Katul, H. S. Horn, S. M. Thomas, R. Oren, R. Avissar, S. W. Pacala \& S. A. Levin. 2002a. Mechanisms of long-distance dispersal of seeds by wind. Nature, 377: $616 \sim 618$.

Nathan, R., H. S. Horn, J. Chave \& S. A. Levin. 2002b. Mechanistic models for tree seed dispersal by wind in dense forests and open landscapes. In: Levey, D. J., W. R. Silva \& M. Galetti eds. Seed dispersal and frugivory: ecology, evolution 
and conservation. New York: CABI Publishing. $69 \sim 82$.

Nathan, R., U. N. Saferiel, I. Noy-Meir \& G. Schiller. 2000. Spatiotemporal variation in seed dispersal and recruitment near and far from Pinus halepensis trees. Ecology, 81:2156 2169 .

Nathan, R., U. N. Safriel \& I. Noy-Meir. 2001. Field validation and sensitivity analysis of a mechanistic model for tree seed dispersal by wind. Ecology, 82:374 388 .

Neubert, M. G. \& H. Caswell. 2000. Demography and dispersal: calculation and sensitivity analysis of invasion speed for structured populations. Ecology, 81: $1613 \sim 1628$.

Norberg, R.A. 1973. Autorotation, self-stability, and structure of single-winged fruits and seeds (samaras) with comparative remarks on animal flight. Biological Reviews, 48:561 $\sim 596$.

Oke, T. R. 1987. Boundary layer climate. London: Methuen Press. $28 \sim 55$.

Okubo, A. \& S. A. Levin. 1989. A theory framework for data analysis of wind dispersal of seeds and pollen. Ecology, 70:329 $\sim 338$.

Opdam, P. 1990. Dispersal in fragmented populations: the key to survival. In: Bruce, R. \& D. Howard eds. Species dispersal in agricultural habitats. London: Belhaven Press. $3 \sim 7$.

Pitelka, L. F., R. H. Gardner, J. Ash, S. Berry, H. Gitay, I. R. Noble, A. Saunders, R. H. W. Bradshaw, L. Brubaker, J. S. Clark, M. B. Davis, S. Sugita, J. M. Dyer, R. Hengeveld, G. Hope, B. Huntley, G. A. King, S. Lavorel, R. N. Mack, G. P. Malanson, M. McGlone, I. C. Prentice \& M. Rejmanek. 1997. Plant migration and climate change. American Scientist, 85:464 473 .

Ridley, H.N. 1930. The dispersal of plants throughout the world. Ashford, English: Reeve Press. $12 \sim 39$.

Shigesada, N., K. Kawasaki \& Y. Takenda. 1995. Modeling stratified diffusion in biological invasions. American Naturalist, 146: $229 \sim 251$.

Shimida, A. \& S. Ellener. 1984. Coexistence of plant species with similar niches. Vegetatio, 58:29 55.

Silvertown. J. \& D. Charlesworth. 2001. Introduction to plant population biology. Oxford: Blackwell Press. $157 \sim 161$.

Skellam, J. G. 1951. Random dispersal in theoretical population. Biometrika, 38:196 218 .

Solbreck, C. \& D. Andersson. 1987. Vertical distribution of fire weed, Epilobium angustifolium, seeds in the air. Canadian Journal of Botany, 65:2177 2178 .
Sorensen, A.E. 1986. Seed disperal by adhesion. Annual Review of Ecology and Systematics, 17:443 463 .

Tackenberg, O. 2003. Modeling long-distance dispersal of plant diasporas by wind. Ecological Monograph, 73:173 189 .

Tackenberg, O., P. Poschlod \& S. Bonn. 2003. Assessment of wind dispersal potential in plant species. Ecological Monograph, 73: $191 \sim 205$.

Turchin, P. 1998. Quantitative analysis of movement: measuring and modeling population redistribution in animal and plants. Surderland: Sinauer Associates. $112 \sim 123$.

van der Pijl, L. 1982. Principle of dispersal in higher plants. Berlin: Springer Press. $454 \sim 470$.

van Dorp, D., W. P. M. van den Hoek \& C. Daleboudt. 1996. Seed dispersal capacity of six perennial grassland species measured in a wind tunnel at varying wind speed and height. Canadian Journal of Botany, 74:1956 1963.

Vander Wall, S.B. \& J.W. Joyner. 1998. Secondary dispersal by the wind of winged pine seed across the ground surface. American Midland Naturist, 139:365 373 .

Walklate, P.J. 1987. A random walk model for dispersion of heavy particles in turbulent air flow. Boundary Layer Meteorology, 39: $175 \sim 190$.

Waser, N.M., R. K. Vickery \& M. V. Price. 1982. Patterns of seed dispersal and populations differentiation in Mimulus guttatus. Evolution, 36:753 761 .

Wieringa, J. 1993. Representative roughness parameters for homogeneous terrain. Boundary-Layer Meteorology, 63:323 363 .

Wilkinson, D. M. 1997. Plant colonization: are wind dispersed seeds really dispersed by birds at large spatial and temporal scales? Journal of Biogeography, 24:61 65.

Willson, M. F. \& A. Traveset. 2000. The ecology of seed dispersal. In: Fenner, M. ed. Seed: the ecology of regeneration in plant communities. New York: CABI Publishing. 85 110 .

Willson, M. F. 1993. Dispersal mode, seed shadows, and colonization patterns. Vegetation, 106/107:261 280 .

Wilson, J. D. \& B. L. Sawford. 1996. Review of Lagarangian stochastic models for trajectories in the turbulent atomosphere. Boundary Layer Meteorology, 78:191 210 .

Yasuda, K. \& A. Azuma. 1997. The autorotation boundary in the flight of samaras. Journals of Theoretical Biology, 185: 313 320 . 